\title{
COMPARATIVO DE NIVELES DE AFRECHO DE YUCA EN RACIONES PARA CERDOS EN CRECIMIENTO Y ENGORDE, EN LA ZONA DE PUCALLPA
}

\author{
J. Rosa1es* \\ H. Urbietta **
}

\section{RESUMEN}

La alimentación representa el $70 \%$ de los costos de crianza de cerdos. Una de las alternativas para disminuir este porcentaje es la utilización de insumos regionales de menos costo, como el afrecho de yuca. El presente estudio se realizó en el Centro Regional de Investigación del IIAP, Pucallpa. El objetivo fue determinar el efecto biológico y económico de niveles crecientes de afrecho de yuca en la ración de cerdos durante las fases de crecimiento y engorde. Se trabajó con tres tratamientos: T1 (10\% de ahecho de yuca), T2 (35\% de afrecho de yuca) y T3 (60\% de afrecho de yuca), durante 98 días. Se evaluó la ganancia de peso, consumo de alimento, conversión alimenticia, y beneficio económico. Los resultados de ganancia de peso (g/animal/día) fueron: $\mathrm{T} 1=684 ; \mathrm{T} 2=621$ y $\mathrm{T} 3=630$ en la fase de crecimiento y $\mathrm{T}=787 ; \mathrm{T} 2=698$ y $\mathrm{T} 3=721$ en la fase de engorde. Estas diferencias no fueron significativas. Igualmente no hubo diferencias entre tratamiento en consumo de alimento diario, siendo en promedio: $\mathrm{T} 1=2,09$; $\mathrm{T} 2=2,06$ y $\mathrm{T} 3=2,03$ $\mathrm{kg} / \mathrm{animal}$ en la fase de crecimiento, y $\mathrm{T} 1=3,11 ; \mathrm{T} 2=2,94$ y T3 $=2,83 \mathrm{~kg} / \mathrm{animal}$ en la fase de engorde. Con referencia a la conversión alimenticia el comportamiento fue similar siendo ligeramente mejor en T1 $(3.54)$, seguido por T3 $(3,59)$ y T2 $(3,79)$. Económicamente el mayor beneficio neto fue en T3, seguido por T2 y T1. Se concluye que la utilización de afrecho de yuca hasta niveles de $60 \%$, no afectaron la ganancia de peso, el consumo de alimento, y la conversión alimenticia; mientras que el $60 \%$ sí trajo consigo mayor beneficio económico.

\footnotetext{
* Investigador del área Pecuaria del Centro Regional de Investigación del Instituto de Investigaciones de la Amazonía Peruana (IIAP) filial Ucayali.

Tesista de la Universidad Naciona1 de Ucayali
} 


\section{INTRODUCCION}

La explotación de cerdos en la zona de Pucallpa es una actividad de baja rentabilidad, debido principalmente a los altos costos de los insumos alimenticios tradicionales para preparar los alimentos balanceados. La alimentación representa $70 \%$ de los costos directos de producción. Una de las alternativas para disminuir estos costos es buscar insumos no tradicionales de bajo costo. Así, durante el proceso de industrialización de la yuca para la producción de harina se obtiene un subproducto llamado "afrecho' que es un insumo energético que podría remplazar parcial o totalmente al maíz en la dieta; por Consiguiente, disminuir los costos de alimentación y hacer más rentable la explotación porcina.

El objetivo de este estudio es determinar el efecto biológico y económico de las proporciones crecientes de afrecho de yuca en las raciones de cerdos durante las fases de crecimiento y engorde.

\section{REVISION DE LITERATURA}

Ríos (1973) afirma que el afrecho de yuca, debido a su bajo costo y buen sabor, se puede usar como fuente energética para la alimentación de vacunos de leche, cerdos, y aves; como suplemento proteico, graso y mineral.

El afrecho y la harina de yuca, en términos de contenido de nutrientes, son similares. Diferenciándose ligeramente el afrecho de yuca por su mayor contenido de proteína y fibra cruda, y por su menor proporción de extracto no nitrogenado (Rosales, 1993).

El afrecho de yuca se usa remplazando parcialmente a otros insumos energéticos tales como sorgo, maíz, y afrecho de trigo. Así, Méndez y Zaragoza (1980) encontraron que la harina de yuca podía remplazar parcialmente, hasta el $72.1 \%$, al sorgo, en raciones de cerdos de cruces comerciales en crecimiento y engorde. Este reemplazo no afectó el rendimiento, la eficiencia de conversión alimenticia, ni las características de la carcasa.

En Tingo María, Bravo (1978) empleando el afrecho de yuca como sustituto del maíz en proporciones de $0,15,30,45$ y $60 \%$ en cerdos en crecimiento, la ganancia de peso diario fue: $610,650,670,650$, y 620 g. y la conversión alimenticia de $2.54 ; 2.43,2.17,2.47$ y 2.37 ; en el análisis no se encontró diferencias $(\mathrm{P}<0.05)$.

En otro estudio en Tingo María en engorde de cerdos, Baldeón (1976) usó $0,9,18,27$ y $36 \%$ de afrecho de yuca en reemplazo parcial del maíz, encontrando que la mejor ganancia de peso y consumo de alimento fue con el $18 \%$ de afrecho de yuca en remplazó del maíz. 
Sin embargo, la conversión alimenticia fue similar en todos los tratamientos. En la evaluación económica, el remplazo del $36 \%$ de afrecho de yuca fue el que produjo mayor rendimiento económico.

Por otro lado, Chicco et al (1972) y Sonaiya et al (1982) afirman que la harina de yuca remplaza parcialmente al maíz amarillo hasta el $45 \%$, en raciones de cerdos en crecimiento y engorde, sin afectar el rendimiento de peso, eficiencia alimenticia, y calidad de la canal. Sin embargo, Shimada, mencionado por Maner y Pond (1974), determinó, usando el 0, 22, 44, y 66\% de harina de yuca en remplazo del maíz, que el nivel máximo de utilización de la harina de yuca fue $44 \%$ y que la ración con $66 \%$ ocasionó una reducción tanto de la ganancia de peso como de la conversión alimenticia.

Barbaosa et al (1979) afirman que al sustituir parcialmente el afrecho de trigo por el afrecho de yuca en raciones de cerdos en crecimiento, no se encontró diferencias en ganancia de peso, consumo de alimento, y eficiencia alimenticia.

\section{MATERIALES Y MÉTODOS}

\section{UBICACIÓN}

El estudio se realizó en la Estación Experimental "Villa Rica" del Centro Regional de Investigación del IIAP, filial Ucayali; ubicada en el Km. 12.4 de la carretera Federico Basadre (Pucallpa-Lima). La estación geográficamente se ubica a 8 22'13" S, 74 34'23" O, y a una altitud de $154 \mathrm{msnm}$.

\section{CONDICIONES CLIMÁTICAS}

La zona pertenece al ecosistema mayor de bosque tropical semisiempreverde estacional (Cochrane, 1982). La precipitación media anual es de 1852 $\mathrm{mm}$, la temperatura media de $25,5 \mathrm{C}$ y la humedad relativa de $82 \%$.

\section{INS TALACIONES Y EQUIPOS}

Se usó una porqueriza de $24 \mathrm{~m} 2$ construida sobre un estanque piscícola, con techo de hojas de palmera, piso y cerco enrejillado de madera, dividida en tres corrales de $6 \mathrm{~m}^{2}$ cada uno $(2 \times 3 \mathrm{~m})$. Cada corral fue equipado con comedero de madera y un bebedero de neumático usado, partido por la mitad. 


\section{ANIMALES}

Se utilizó nueve gorrinos cruzados de las razas Landrace por Yorkshire $(1 / 2$ x $1 / 2$ aproximadamente), cinco machos castrados y cuatro hembras, con una edad de 11 semanas y con un peso promedio de $22 \mathrm{~kg}$. Los nueve gorrinos fueron distribuidos indistintamente hembras y machos en grupos de tres animales. Cada grupo fue alojado en un corral.

\section{TRATAMIENTOS}

Cada grupo de animales fue alimentado con una de tres raciones isoproteícas e isoenergéticas, pero con diferentes proporciones de afrecho de yuca, los que dieron lugar a tres tratamientos:

$\mathrm{T} 1$ = Ración con $10 \%$ de afrecho de yuca en la ración.

T2 = Ración con 35\% de afrecho de yuca en la ración.

T3 = Ración con $60 \%$ de afrecho de yuca en la ración.

\section{ALIMENTACION}

Se estableció dos etapas de crianza: la de crecimiento de 11 a 18 semanas y la de engorde de 10 a 25 semanas de edad. Las fórmulas de las tres dietas y las especificaciones nutricionales tanto de las raciones de crecimiento como la de engorde se presenta en los cuadros 1 y 2 . El análisis proximal de los alimentos se muestra en el cuadro 3.

Los cerdos se alimentan dos veces al día: la primera a 08:00 y la segunda a 13:00. El suministro de agua fue ad libitum con agua de la propia piscigranja. 


\section{Cuadro 1}

FÓRMULA DE LAS TRES RACIONES EXPERIMENTALES PARA CERDOS EN LA FASE DE CRECIMIENTO Y ENGORDE

\begin{tabular}{|c|c|c|c|c|c|c|}
\hline \multirow{3}{*}{ Insumos } & \multicolumn{6}{|c|}{ Tratamiento } \\
\hline & \multirow[b]{2}{*}{ TI } & \multicolumn{3}{|c|}{ Crecimiento } & \multicolumn{2}{|c|}{ Engorde } \\
\hline & & $\mathrm{T} 2$ & $\mathrm{~T} 3$ & $\mathrm{~T} 1$ & $\mathrm{~T} 2$ & $\mathrm{~T} 3$ \\
\hline Afrecho de yuca & 10,0 & 35,0 & 60,0 & 10,0 & 35,0 & 60,0 \\
\hline Maíz amarillo & 37,0 & 8,0 & 4,5 & 45,0 & 16,0 & 12,5 \\
\hline Polvillo de arroz & 29,0 & 29,0 & 5,0 & 29,0 & 29,0 & 5,0 \\
\hline Hna.de pescado II & 23,0 & 27,0 & 29,0 & 15,0 & 19,0 & 21,5 \\
\hline Sal mineral & 0,5 & 0,5 & 0,5 & 0,5 & 0,5 & 0,5 \\
\hline Sal común & 0,5 & 0,5 & 0,5 & 0,5 & 0,5 & 0,5 \\
\hline Total & 100,0 & 100,0 & 100,0 & 100,0 & 100,0 & 100,0 \\
\hline
\end{tabular}

\section{Cuadro 2}

ESPECIFICACIONES NUTRICIONALES EN LA FORMULACIÓN DE LAS RACIONES PARA CERDOS EN LA FASE DE CRECIMIENTO Y ENGORDE (CALCULADOS).

\begin{tabular}{|c|c|c|c|c|c|c|}
\hline \multirow{3}{*}{ Nutrientes } & \multicolumn{6}{|c|}{ Tratamiento } \\
\hline & \multicolumn{2}{|c|}{ Crecimiento } & \multicolumn{4}{|c|}{ Engorde } \\
\hline & TI & $\mathrm{T} 2$ & $\mathrm{~T} 3$ & $\mathrm{~T} 1$ & $\mathrm{~T} 2$ & $\mathrm{~T} 3$ \\
\hline $\mathrm{ED}^{1}(\mathrm{Mcal} / \mathrm{kg})$ & 3,15 & 3,00 & 3,01 & 3,16 & 3,03 & 3,00 \\
\hline $\mathrm{EE}^{2}(\%)$ & 6,23 & 5,16 & 4,45 & 5,69 & 4,61 & 3,76 \\
\hline Proteína (\%) & 16,08 & 16,07 & 16,04 & 13,06 & 13,05 & 13,03 \\
\hline Fibra $\quad(\%)$ & 6,99 & 7,86 & 5,19 & 7,11 & 8,00 & 6,65 \\
\hline Grasa (\%) & 6,23 & 5,16 & 4,45 & 5,69 & 4,61 & 3,76 \\
\hline $\mathrm{Ca} \quad(\%)$ & 1,93 & 2,26 & 2,46 & 1,33 & 1,66 & 1,87 \\
\hline$(\%)$ & 1,00 & 1,15 & 1,22 & 0,71 & 0,85 & 0,92 \\
\hline
\end{tabular}

$1=$ Energía Digestible

$2=$ Extracto Etereo 


\section{Cuadro 3}

ANÁLISIS PROXIMA ${ }^{1}$ DE LAS RACIONES PARA CERDOS EN LA FASE DE CRECIMIENTO Y ENGORDE (TAL COMO OFRECIDO)

\begin{tabular}{|c|c|c|c|c|c|c|}
\hline \multirow{3}{*}{ Nutrientes } & \multicolumn{6}{|c|}{ Tratamientos } \\
\hline & \multicolumn{2}{|c|}{ Crecimiento } & \multicolumn{4}{|c|}{ Engorde } \\
\hline & $\mathrm{T} 1$ & $\mathrm{~T} 2$ & $\mathrm{~T} 3$ & $\mathrm{~T} 1$ & $\mathrm{~T} 2$ & $\mathrm{~T} 3$ \\
\hline Humedad & 9,00 & 11,55 & 11,60 & 11,57 & 11,25 & 11,95 \\
\hline Proteína & 16,88 & 16,04 & 14,78 & 13,98 & 13,14 & 12,75 \\
\hline Grasa & 6,00 & 5,10 & 2,50 & 5,59 & 4,70 & 3,85 \\
\hline Fibra & 4,20 & 5,73 & 5,43 & 6,55 & 7,35 & 5,98 \\
\hline Ceniza & 9,00 & 11,55 & 11,60 & 9,25 & 10,68 & 10,46 \\
\hline Nifex & 54,92 & 50,03 & 54,09 & 53,06 & 52,88 & 55,01 \\
\hline TOTAL & 100,00 & 100,00 & 100,00 & 100,00 & 100,00 & 100,0 \\
\hline
\end{tabular}

1/ Laboratorio de Nutrición Animal de la Universidad Nacional Agraria, La Molina.

\section{SANIDAD}

Antes del inicio del experimento los animales fueron vacunados contra el cólera porcino, cepa china, virus vivo modificado. Además, tanto al inicio del período de crecimiento como al del período de engorde fueron dosificados contra parásitos gastrointestinales con clorhidrato de Levamisol (Prolevan inyectable) y bañados contra parásitos externos con Hexaclorociclohexano (Sarnavet).

\section{VARIABLES MEDIDAS}

\section{Consumo de alimento}

El control del consumo de alimento se realizó diariamente, calculando la diferencia entre el alimento ofrecido y el residual y el desperdicio; de esta manera se obtuvo el consumo diario. 


\section{Ganancia de peso}

El control de peso se realizó semanalmente durante todo el experimento. Los pesos fueron tomados en una balanza tipo plataforma de $500 \mathrm{~kg}$. de capacidad con una aproximación de $250 \mathrm{~g}$.

\section{Conversión alimenticia}

La cantidad de alimento consumido por el animal para ganar un kilo de peso vivo.

\section{Análisis económico}

Para el análisis económico se tuvo en cuenta los costos fijos y variables. La ecuación usada para el cálculo del beneficio neto fue la siguiente:

$$
\mathrm{Bi}=\mathrm{PYi}-(\mathrm{CVi}+\mathrm{CF})
$$

Donde:

$\begin{array}{lll}\mathrm{Bi} & = & \text { Beneficio neto, en nuevos soles, por cerdo } \\ \mathrm{i} & = & \text { Tratamientos } \\ \mathrm{P} & = & \text { Precio por kilogramo de cerdo, en nuevos soles } \\ \mathrm{Yi} & = & \text { Peso promedio por cerdo, por tratamiento } \\ \mathrm{CVi} & = & \text { Costo variable por cerdo, por tratamiento } \\ \mathrm{CF} & = & \text { Costo fijo por cerdo, por tratamiento }\end{array}$

\section{DISEÑO ESTADÍSTICO}

Se usó el diseño Completamente al Azar, con tres repeticiones (Calzada, 1982), siendo el Modelo Aditivo Lineal:

$$
Y i j=u+t i+E i j
$$

Donde:

Yij $=\quad$ Valor observado para la j-ésima repetición del tratamiento i-ésimo.

$\mathrm{u}=\quad$ Media poblacional 
ti $=$ Efecto del i-ésimo tratamiento

Eij $\quad=\quad$ Error experimental

\section{RESULTADOS Y DISCUSION}

\section{CONSUMO DE ALIMENTO}

El consumo de alimento diario, tal como el ofrecido, durante la fase de crecimiento y engorde se presenta en el cuadro 4 . Se observa que no existen mayores diferencias entre tratamientos. Se puede atribuir que estos resultados obedecen a lo siguiente: raciones isoenergéticas, alimento con volumen similar, buen sabor de las raciones y la utilización de buena fuente proteica.

Resultados ligeramente menores de consumo de alimento, pero que no difieren $(\mathrm{P}<0.05)$, fueron reportados por Méndez y Zaragoza (1980) en cerdos en crecimiento de 19 a $50 \mathrm{~kg}$, donde el consumo diario fue 1,$92 ; 1,93 ;$ y $1,93 \mathrm{~kg}$ para los tratamientos con $0 ; 35,8$; y $67 \%$ de harina de yuca en la ración, respectivamente. Sin embargo, en engorde el consumo diario fue mayor: 3,$17 ; 3,44$; y 3,24 kg para los tratamientos de $0 ; 39 ;$ y 72,1 \% de harina de yuca en la ración, respectivamente. Estas diferencias podrían deberse: a la raza de animal, insumos alimenticios usados, manejo animal, y factores climáticos.

\section{Cuadro 4}

CONSUMO DE ALIMENTO DIARIO, TAL COMO EL OFRECIDO, DE CERDOS YORKSHIRE X LANDRACE ${ }^{1}$ EN CRECIMIENTO Y ENGORDE, EN UN PERÍODO DE 98 DÍAS.

Fase

Tratamiento

$\mathrm{T} 1$

$\mathrm{T} 2$ T3

g/animal/día

Crecimiento

2,09

2,06

2,03

Engorde

3,11

2,94

2,83

1/ Cerdos de 11 semanas de edad al inicio del experimento

2/ Afrecho de yuca en la ración 


\section{GANANCIA DE PESO}

Los pesos iniciales y finales, así como la ganancia de peso durante la fase de crecimiento y la de engorde se los muestra en el cuadro 5. No se encontró diferencias $(\mathrm{P}<0,05)$ en la ganancia de peso tanto en la fase de crecimiento como en la de engorde.

En el presente estudio se usó harina de pescado para compensar las diferencias proteicas, vitamínicas, y de minerales entre el afrecho y el maíz; haciendo las raciones isoproteícas e isoenergéticas. Esto posiblemente influyó a que no se presenten diferencias estadísticas.

Ganancias menores a las encontradas, pero con igual tendencia; vale decir, que no difieren entre tratamientos fueron informados por Sonaiya et al. (1982) quienes usando $0,15,30$ y $45 \%$ de harina de yuca en la ración de cerdos Large White en crecimiento y engorde encontraron aumentos de peso diarios de 390; 420; 460; y 430 g/animal, respectivamente. También, Méndez y Zaragoza (1980), trabajando con cerdos de cruces comerciales encontraron ganancias de peso (g/animal/día) de 560; 540; 570; y 550 para raciones que contenían $0 ; 18,6 ; 35,8$; 52,$0 ;$ y $67,0 \%$ de harina de yuca, respectivamente, durante la fase de crecimiento. Las ganancias de peso encontradas (g/animal/día) durante la fase de engorde fueron 740; 720 790; 730; y 710 para dietas con $0 ; 20 ; 39 ; 56,5 ;$ y 72,1 \% de harina de yuca en la ración, respectivamente.

\section{Cuadro 5}

PESO INICIAL, FINAL Y GANANCIA DIARIA DE PESO DE CERDOS YORKSHIRE LANDRACE ${ }^{1}$ EN CRECIMIENTO Y ENGORDE, EN UN PERÍODO DE 98 DÍAS. PROMEDIO DE TRES REPETICIONES

\begin{tabular}{llllll}
\hline & \multicolumn{3}{c}{ Peso Final } & \multicolumn{2}{c}{ Ganancia diaria } \\
Tratamiento & Peso inicial & Crecimiento & Engorde & Crecimiento & Engorde \\
\hline \multirow{2}{*}{ T1 } & 2960 & 56,13 & 94,70 & 684 & 787 \\
T2 & 20,70 & 51,13 & 85,33 & 621 & 698 \\
T3 & 22,80 & 53,67 & 89,00 & 630 & 721 \\
\hline \multirow{2}{*}{ Promedio } & 22,03 & 53,64 & 89,68 & 645 & 735 \\
\hline
\end{tabular}

1/ Cerdos de II semanas de edad al inicio del experimento. 


\section{CONVERSION ALIMENTICIA}

La conversión alimenticia durante el período de crecimiento y engorde se muestra en el cuadro 6. Se observa que la ración con $10 \%$ de afrecho de yuca fue la mejor conversión alimenticia, pero esta superioridad no fue significativa.

\section{Cuadro 6}

Conversión alimenticia de cerdos Yorkshire $\mathrm{x}$ Landrace $^{1}$ en un período de 98 días.

Fase

Tratamientos

$\mathrm{T} 1 \quad \mathrm{~T} 2$

T3

\begin{tabular}{llcc} 
Crecimiento & 3,06 & 3,32 & 3,22 \\
Engorde & 3,95 & 4,21 & 3,92 \\
Promedio & 3,54 & 3,79 & 3,59 \\
\hline
\end{tabular}

1/ Cerdos de 11 semanas de edad al inicio del experimento.

2/ Afrecho de yuca en la ración.

Méndez y Zaragoza (1980) en un estudio similar, con cerdos de cruces comerciales en crecimiento y engorde, encontraron resultados con menor eficiencia alimenticia, pero con igual tendencia a lo encontrado. Los resultados fueron 3,46; 3,61 , y 3,55 para las raciones con $0 ; 35,8$; y $67 \%$ de harina de yuca, durante la fase de crecimiento; y 4,29; 4,56 y 4,6 para las raciones con $0 ; 39$ y 72,1\% de harina de yuca, durante la fase de engorde, respectivamente.

\section{ANALISIS ECONÓMICO}

Para el análisis económico se usó los costos fijos y los costos variables, tal Como se indica en los cuadro 7 y 8 , respectivamente. El costo se realizó al cambio de S/. 0.96 por dólar. 


\section{Cuadro 7}

COSTO VARIABLES POR CERDO Y POR TRATAMIENTO

Variables Tratamientos

$\mathrm{T} 1$ $\mathrm{T} 2$ T3

Consumo de alimento:

Crecimiento (kg/animal) 102,56

101,01

99,28

Engorde (kg/animal) $\quad 152,38$

144,03

138,43

Costo del alimento:

Crecimiento $(\mathrm{S} / . / \mathrm{Kg})$

0,26

0,22

0,22

Engorde $(\mathrm{S} / . / \mathrm{Kg})$

0,24

0,20

0,18

Costo de alimentación :

Crecimiento (S/.)

26,67

2999

21,84

Engorde (S/.)

36,57

28,81

24,92

COSTO TOTAL DE
ALIMENTACION (S/.) 63,24
51,03
46,76

\section{Cuadro 8}

COSTO FIJO POR CERDO Y POR TRATAMIENTO

Parámetros

$$
\text { T1 }
$$

Gorrino

Sanidad

Mano de obra

TOTAL
Tratamientos

$\mathrm{T} 2$

T3

29,64

1,89

3,00 
El análisis económico que se obtuvo al final del estudio se muestra en el cuadro 9, donde se observa una utilidad neta, por kilo de carne, de soles 0,268; 0,329 y 0,387 para los tratamientos $\mathrm{T} 1, \mathrm{~T} 2$ y $\mathrm{T} 3$, respectivamente. Lo que demuestra que con la ración con $60 \%$ de afrecho de yuca se obtuvo ventajas económicas mayores, entre 15 y $30 \%$, respecto a los tratamientos T2 y T1, respectivamente. Esta ventaja económica, se debería a que el costo del alimento de las raciones del T3 y T2 son más económicas, por tener dentro de su composición mayores proporciones de afrecho de yuca, que es un insumo más económico que el maíz.

\section{Cuadro 9}

BENEFICIO PROMEDIO NETO POR ANIMAL POR TRATAMIENTO EN NUEVOS SOLES.

\begin{tabular}{|c|c|c|c|c|c|c|c|}
\hline Trat. & $\mathrm{P}$ & $\mathrm{Y}(\mathrm{kg})$ & PxY & $\mathrm{CV}$ & $\mathrm{CF}$ & $\begin{array}{l}\mathrm{B} / \\
\text { Animal }\end{array}$ & $\begin{array}{l}\mathrm{B} / \mathrm{kg} \\
\text { Carne }\end{array}$ \\
\hline
\end{tabular}

\begin{tabular}{llllllll} 
T1 & 1,80 & 94,70 & 123,11 & 63,24 & 34,53 & 25,34 & 0.368 \\
T2 & 1,80 & 85,33 & 110,93 & 51,03 & 31,80 & 28,10 & 0.329 \\
T3 & 1,80 & 89,00 & 115,70 & 46,76 & 34.53 & 34,41 & 0387 \\
\hline
\end{tabular}

\section{CONCLUSIONES}

De los resultados obtenidos en el presente estudio, se derivan las siguientes conclusiones:

- La utilización del afrecho de yuca hasta el $60 \%$ de la ración, no afectó significativamente la ganancia de peso, el consumo de alimento, y la eficiencia alimenticia.

- El mayor beneficio económico se obtuvo con $60 \%$ de afrecho de yuca, seguido por los tratamientos con 35 y $10 \%$ de afrecho de yuca. 


\section{BIBLIOGRAFIA}

BALDEOS, R. 1976. "Utilización de varios niveles de afrecho de yuca en el engorde de cerdos”. Tesis. Ing. Zootecnista, Universidad Nacional Agraria de la Selva Tingo María. $62 \mathrm{p}$.

BARBOSA, S. et al 1979. "A raspa de mandioca como substituto dos subproductos de trigo no crecimiento de suinos”. Arq. Ese. Supr. Vet (Brasil) 10:15-24.

BUITRAGO, J.A. 1990. La yuca en la alimentación animal. Centro Internacional de Agricultura Tropical. Cali, Colombia, 446 p.

CALZADA, J. 1982. Métodos estadísticos para la investigación. 5 ed,, Editorial Milagros S.A., Lima, Perú. 644 p.

CHICCO, C.F.; GARBATTI, S.T.; MULLER-MAYE, E.; VECCHIONACCE, H. 1972. "La harina de yuca en el engorde de cerdos", en Agronomía Tropical, Venezuela, 22(6) : 599-603.

COCHRANE, T.T. 1982. "Caracterización agroecológica para el desarrollo de pasturas en suelos ácidos de América Tropical”, en Toledo, J.M. (ed); Manual para la Evaluación Agronómica. Red Internacional de Pastos Tropicales, Cali, Colombia, CIAT. pp. 23- 44.

MANER, H.; POND, 0. 1974. Producción de cerdos en climas templados y tropicales. Editorial Acribia. Zaragoza. España.

MENDEZ, A.; ZARAGOZA, L. 1980. "Sustitución del sorgo por harina de yuca en la alimentación de cerdos", en Agrie. Tec. Mex. 6(2): 83-9 1.

RIOS, B. 1973. "Digestibilidad del afrecho de yuca en ovinos". Tesis Ing. Zootecnista. Universidad Nacional Agraria de la Selva, Tingo María. 41 p.

ROSALES, J.M. 1993. "Valor nutritivo de insumos regionales en alimentación animal". Informe Técnico Interno. Centro Regional de Investigación del Instituto de Investigaciones de la Amazonía Peruana, Pucallpa. 10 p.

SONAIYA, E.B.; OMOLE, T.A.; ADEGBOLA, A.A. 1982. "Effects of methionine suplemented cassava meal diets on performance and carcass characteristics and some organ weights of growing finishing pigs", en Nutrition Reports International 26(1):25-34. 